\title{
RESPUESTA DE ZARZAMORA (Rubus spp.) CV. TUPY A LA SALINIDAD
}

\author{
RESPONSE OF BLACKBERRY (Rubus spp.) \\ CV. TUPY TO SALINITY
}

\section{Sandra L. González-Jiménez, Ana Ma. Castillo-González, Ma. del Rosario García- Mateos $^{1}$, Luis A. Valdez-Aguilar ${ }^{2}$, Carmen Ybarra-Moncada ${ }^{3}$ y Edilberto Avitia-García ${ }^{1}$}

\begin{abstract}
'Universidad Autónoma Chapingo (UACh), Departamento de Fitotecnia, Chapingo, Estado de México. México. ${ }^{2}$ Universidad Autónoma Agraria Antonio Narro, Departamento de Horticultura, Buenavista, Saltillo, Coahuila, México. ${ }^{3}$ UACh, Instituto de Alimentos, Chapingo, Estado de México, México.

*Autor de correspondencia (anasofiacasg@hotmail.com)
\end{abstract}

\section{RESUMEN}

La zarzamora (Rubus spp.) es un cultivo de importancia socioeconómica en México y sus frutos tienen alto valor nutricional; sin embargo, existe poca información del efecto de la salinidad en este cultivo; por ello, el objetivo del presente estudio fue determinar el efecto de la salinidad en el crecimiento, rendimiento, concentración de prolina en la raíz y calidad del fruto de zarzamora Tupy. Se establecieron plantas en macetas de $19 \mathrm{~L}$ con tezontle $(2 \mathrm{a} 3 \mathrm{~mm})$ y se regaron con solución nutritiva mediante un sistema hidropónico abierto. Los tratamientos fueron soluciones nutritivas salinas con conductividades eléctricas (CE) de 2.0 (testigo), 2.2, 2.4, 2.6, 2.8 y $3.0 \mathrm{dS} \mathrm{m}^{-1}$, aplicadas diariamente desde las 45 semanas después del trasplante hasta la fructificación. El rendimiento, peso seco de planta, diámetro de caña, número de frutos, concentración de clorofila total y carotenoides se redujeron en 60 , $30,24,54,50$ y $46 \%$, respectivamente, y la concentración de prolina en la raíz se incrementó en $122 \%$ con $3.0 \mathrm{dS} \mathrm{m}^{-1}$. Las conductividades de 2.6 a $3.0 \mathrm{dS}$ ocasionaron disminución en el peso, largo y diámetro del fruto en 16, 11 y 6.9 $\%$, respectivamente; el índice de redondez fue $6 \%$ menor con $3.0 \mathrm{dS}$ y sólo la CE de $2.8 \mathrm{dS} \mathrm{m}^{-1}$ elevó la firmeza en $11 \%$; los demás tratamientos y el testigo tuvieron valores estadísticamente similares. Con $3.0 \mathrm{dS} \mathrm{m}^{-1}$ los azúcares solubles totales del fruto disminuyeron $50 \%$, la acidez titulable y los sólidos solubles totales se incrementaron en 22 y $8 \%$, la concentración de fenoles y flavonoides fue 25 y $29 \%$ menor; mientras que la capacidad antioxidante y el porcentaje de inhibición fueron 21 y $18 \%$ mayores respectivamente. La concentración de antocianinas fue $95 \%$ mayor con conductividades de 2.2 a $2.8 \mathrm{dS}$.

Palabras clave: Rubus spp., antocianinas, capacidad antioxidante, prolina, rendimiento, vitamina C.

\section{SUMMARY}

Blackberry (Rubus spp.) is a crop of socioeconomic importance in Mexico, and its fruits have high nutritional value. However, there is little information on the effect of salinity on this crop, so this research focused on the impact of salinity on growth, yield, proline content in the root, and fruit quality of blackberry cv. Tupy. Plants were sowed in $19 \mathrm{~L}$ pots with tezontle (red volcanic rock) (2 to $3 \mathrm{~mm}$ ) and were watered with a nutrient solution using an open hydroponic system. The treatments were saline nutrient solutions with electrical conductivities (CE) of 2.0 (control), 2.2, 2.4, 2.6, 2.8, and $3.0 \mathrm{dS} \mathrm{m}^{-1}$, applied daily from 45 weeks after transplant until fruiting. Yield, plant dry weight, diameter of the cane, number of fruits, total chlorophyll concentration, and carotenoids content were reduced by $60,30,24,54,50$ and $46 \%$, respectively, and the concentration of proline in the root increased by $122 \%$ with $3.0 \mathrm{dS} \mathrm{m}^{-1}$. Conductivities from 2.6 to $3.0 \mathrm{dS}$ caused a decrease in weight, length, and diameter of the fruit by 16,11 , and $6.9 \%$, respectively. The roundness index was $6 \%$ lower with $3.0 \mathrm{dS}$, and only the $2.8 \mathrm{dS} \mathrm{m}^{-1}$ conductivity increased fruit firmness by $11 \%$. The remaining treatments and control had statistically similar values. With $3.0 \mathrm{dS} \mathrm{m}^{-1}$, total soluble sugars of the fruit decreased by $50 \%$, titratable acidity and total soluble solids increased by $22 \%$ and $8 \%$, the concentration of phenols and flavonoids was 25 and 29 $\%$ lower, while the antioxidant capacity and the inhibition percentage were 21 and $18 \%$ higher respectively. The anthocyanin concentration was $95 \%$ higher, with conductivities of 2.2 to $2.8 \mathrm{dS}$.

Index words: Rubus spp., anthocyanins, antioxidant capacity, proline, vitamin $\mathrm{C}$, yield

\section{INTRODUCCIÓN}

A nivel mundial el $20 \%$ de las tierras cultivadas bajo riego (62 millones de hectáreas) están afectadas por salinidad (Khan et al., 2015). En México se estima que 10 $\%$ de la superficie irrigada está afectada por salinidad y de ésta, el $64 \%$, aproximadamente, se encuentra en la zona norte del país (Ruiz et al., 2007). El estrés salino causa enormes pérdidas en la agricultura, ya sea por efecto de la salinidad (suelos con alta concentración de sales solubles) o sodicidad (suelos con alto porcentaje de $\mathrm{Na}^{+}$ intercambiable) (Munns, 2005). La salinidad es causada por las interacciones de varias sales como cloruro de sodio y sulfato de magnesio en zonas costeras, o de bicarbonato de sodio, cloruro de sodio y sulfato de magnesio en suelos sódicos (Ríos-Gómez et al., 2010). Los efectos perjudiciales de la salinidad en las plantas se engloban en la limitación del crecimiento y la productividad, afectan procesos como la germinación, fotosíntesis y absorción de agua, y causa desequilibrio nutrimental y estrés oxidativo (Parihar et al., 2015).

Las zarzamoras y otras especies del género Rubus tienen una expansión de producción alta debido a la combinación 
de distintos factores, como la creación de cultivares mejorados, mercadeo en ascenso y un aumento en el consumo mundial de frutillas, especialmente como fruta fresca (Clark y Finn, 2014). Después de la fresa (Fragaria $x$ ananassa), la zarzamora se ha colocado como la segunda frutilla más producida en México (FIRA, 2016). En 2019 la producción nacional fue de 297,485 t (SIAP, 2020); los principales estados productores son Michoacán, Jalisco y Colima, y los cultivares predominantes son Brazos y Tupy, que se consideran tipos semierectos (Strik et al., 2007).

La zarzamora cuenta con propiedades antioxidantes debido a cantidades altas de compuestos fenólicos, principalmente antocianinas (Zhao, 2007). Al igual que otras frutillas, ha sido considerada como sensible a conductividades eléctricas altas, pues a $25{ }^{\circ} \mathrm{C}$, la conductividad de $2.5 \mathrm{dS} \mathrm{m}^{-1}$ puede reducir el rendimiento del 10 al $25 \%$ (Kotuby-Amacher et al., 2000). Algunos autores han evaluado el efecto del estrés salino en otras especies de frutillas como fresa (Garriga et al., 2015; González-Jiménez et al., 2020; Keutgen y Pawelzik, 2007) y frambuesa (Rubus idaeus, Neocleous y Vasilakakis, 2008), cuyas investigaciones se basaron principalmente en altas dosis de $\mathrm{NaCl}$ como causante de la salinidad; sin embargo, la naturaleza del suelo salino puede ser provocada por la presencia e interacción de otros iones como $\mathrm{Ca}^{2+}, \mathrm{Mg}^{2+}, \mathrm{Cl}^{-}$, $\mathrm{Na}^{+}$, sulfato $\left(\mathrm{SO}_{4}{ }^{2-}\right)$, bicarbonato $\left(\mathrm{HCO}_{3}^{-}\right)$y en algunos casos $\mathrm{K}^{+} \mathrm{y} \mathrm{NO}_{3}{ }^{-}$(Parihar et al., 2015; Ríos-Gómez et al., 2010).

La creciente derrama económica proveniente de la producción de zarzamora en México y el mundo, sus beneficios a la salud y el interés en la proyección de nuevos sitios de cultivo no habituales para esta especie, como zonas tropicales y áridas, son razones suficientes para investigar sobre las relaciones existentes entre el entorno salino y sus efectos en la planta y frutos, por lo que la presente investigación tuvo como objetivos conocer el efecto de la salinidad en el crecimiento, concentración de prolina en la raíz, rendimiento y calidad del fruto de zarzamora cv. Tupy para conocer la posibilidad de su cultivo en suelos con algún grado de salinidad.

\section{MATERIALES Y MÉTODOS}

\section{Sitio experimental}

La investigación se realizó en un invernadero de cristal en la Universidad Autónoma Chapingo, Estado de México ( $19^{\circ} 20^{\prime} \mathrm{N}$ y $98^{\circ} 53^{\prime} \mathrm{O}$ a $2240 \mathrm{~m}$ de altitud); durante todo el periodo de investigación (de noviembre de 2016 a junio de 2018) se registraron la temperatura y la humedad relativa con un data logger $\left(\mathrm{HOBO}{ }^{\circledR}\right.$ U12-012, Burlington, Vermont, EUA); la temperatura promedio durante el experimento fue de $22.7^{\circ} \mathrm{C}$, con una máxima de $42^{\circ} \mathrm{C}$ y una mínima de 8.2
${ }^{\circ} \mathrm{C}$; la humedad relativa media fue de $52.14 \%$. Se utilizaron plántulas de zarzamora cultivar Tupy procedentes de Zamora, Michoacán, las cuales fueron establecidas en macetas de $19 \mathrm{~L}$ de capacidad con tezontle $(2$ a $3 \mathrm{~mm}$ de diámetro) colocadas a $45 \mathrm{~cm}$ de distancia entre macetas y de $90 \mathrm{~cm}$ entre hileras, en un sistema hidropónico abierto.

\section{Tratamientos}

Los tratamientos consistieron en niveles crecientes de salinidad, expresados en términos de conductividad eléctrica (CE): 2.0 (testigo), 2.2, 2.4, 2.6, 2.8 y 3.0 dS m-1. En el Cuadro 1 se presenta la composición de las soluciones nutritivas; los micronutrimentos se abastecieron con $0.035 \mathrm{~g} \mathrm{~L}^{-1}$ de la mezcla comercial TradeCorp ${ }^{\circledR}$ AZ. A las cinco semanas después del trasplante (SDT) las plantas se regaron a diario y manualmente con $1800 \mathrm{~mL}$ de la solución nutritiva de CE de $2.0 \mathrm{dS} \mathrm{m}^{-1}$ (testigo), a las 20 SDT el volumen subió a $2500 \mathrm{~mL}$, a partir de las 40 SDT éste fue de $3000 \mathrm{~mL}$, con drenaje del 25 al $30 \%$. Los tratamientos se aplicaron diariamente a partir de las 45 SDT hasta las 77 SDT (ocho meses de tratamiento).

\section{Diseño y unidad experimental}

El diseño experimental fue completamente al azar con 10 repeticiones; la unidad experimental consistió en una maceta con una planta; sin embargo, para las variables destructivas de planta se seleccionaron al azar cinco plantas por tratamiento, por lo que el número de repeticiones en diversas variables fue de cinco, como se indica posteriormente.

\section{Variables morfológicas y químicas evaluadas}

Al final del experimento, 77 SDT, se evaluaron las siguientes variables:

Peso seco de planta. Se seleccionaron al azar cinco plantas por tratamiento, cada planta se seccionó en parte aérea y raíz, se registró el peso seco con una balanza digital Ohaus ${ }^{\circledR}$ modelo Scout Pro (Parsippany-Troy Hills, New Jersey, EUA); para secar el material se usó una estufa con aire forzado Binder ${ }^{\circledast}$ (Tuttlingen, Alemania) a $65^{\circ} \mathrm{C}$ por $72 \mathrm{~h}$ o hasta peso constante.

Rendimiento por planta. Se cosechó y pesó el total de frutos por planta $(\mathrm{g})$ con una balanza digital Ohaus $^{\circledR}$ modelo Scout Pro (Parsippany-Troy Hills, New Jersey, EUA).

Número de frutos por planta. Se cuantificaron todos los frutos maduros completos (con todas las drupeolas formadas y que alcanzaron un color negro brillante). 
Cuadro 1. Concentración de iones $\left(\mathrm{mmol} \mathrm{L}^{-1}\right)$ de las soluciones nutritivas salinas con diferentes $\mathrm{CE}$ utilizadas en el cultivo hidropónico de zarzamora cv. Tupy.

\begin{tabular}{llllllllllll}
\hline $\mathrm{CE}\left(\mathrm{dS} \mathrm{m}{ }^{-1}\right)$ & $\mathrm{NO}_{3}^{-}$ & $\mathrm{H}_{2} \mathrm{PO}_{4}^{-2}$ & $\mathrm{SO}_{4}^{-2}$ & $\mathrm{HCO}_{3}^{-}$ & $\mathrm{Cl}^{-}$ & $\mathrm{NH}_{4}^{+}$ & $\mathrm{K}^{+}$ & $\mathrm{Ca}^{2+}$ & $\mathrm{Mg}^{2+}$ & $\mathrm{Na}^{+}$ & $\mathrm{pH}$ \\
\hline 2.0 (testigo) & 15.36 & 1.78 & 0.48 & 2.22 & 0.56 & 0.87 & 7.40 & 4.34 & 1.01 & 0.90 & 6.0 \\
2.2 & 15.36 & 1.78 & 1.40 & 2.22 & 0.85 & 0.87 & 7.40 & 4.78 & 1.49 & 1.19 & 6.0 \\
2.4 & 15.36 & 1.78 & 2.06 & 2.22 & 1.48 & 0.87 & 7.40 & 5.24 & 1.69 & 1.82 & 6.0 \\
2.6 & 15.36 & 1.78 & 2.66 & 2.22 & 2.14 & 0.87 & 7.40 & 5.64 & 1.89 & 2.48 & 6.0 \\
2.8 & 15.36 & 1.78 & 3.30 & 2.22 & 2.76 & 0.87 & 7.40 & 6.09 & 2.08 & 3.10 & 6.2 \\
3.0 & 15.36 & 1.78 & 3.96 & 2.22 & 3.38 & 0.87 & 7.40 & 6.52 & 2.28 & 3.72 & 6.2 \\
\hline
\end{tabular}

Contenido de prolina en raíz. Se determinó con el método de Bates et al. (1973); de cinco plantas por tratamiento se pesaron $0.5 \mathrm{~g}$ de muestra fresca de ápices de raíz. Se realizaron lecturas a $520 \mathrm{~nm}$ en un espectrofotómetro ThermoSpectronic ${ }^{\circledR}$ modelo Genesys 10 UV (New York, EUA). La concentración de prolina se estimó a partir de una curva patrón de 0 a $39 \mu_{\mathrm{g} \mathrm{mL}}^{-1}$ de prolina Sigma Aldrich ${ }^{\circledast}$.

Contenido de pigmentos fotosintéticos (PF). Se determinaron las concentraciones de clorofila A (CA), clorofila B (CB) y carotenoides (CAR) como lo indica la AOAC (1980) y la técnica descrita por Witham et al. (1971); para ello, se utilizaron dos hojas de reciente maduración, de cada una de cinco repeticiones por tratamiento. Los resultados se expresaron en $\mathrm{mg} \mathrm{g}^{-1}$ p.f.

Longitud y diámetro de la caña principal. La longitud se midió en cm desde la base hasta la punta de la caña principal con un flexómetro marca Truper $^{\circledR}$ modelo Gripper-5m (China); el diámetro se midió en mm en la base de la misma caña con un vernier digital General ${ }^{\circledR}$ No. 143 (Suiza).

Azúcares solubles totales (AST). Se determinaron con el método de antrona descrito por Witham et al. (1971), se pesaron $2 \mathrm{~g}$ de raíz de la parte media y de la mezcla de cinco hojas de reciente maduración por planta, ésto se hizo en cinco repeticiones por tratamiento. Se realizaron lecturas a $600 \mathrm{~nm}$ en un espectrofotómetro Thermo Spectronic ${ }^{\circledR}$ modelo Genesys 10 UV (New York, EUA). La concentración de azúcares se calculó a partir de una curva patrón que contenía hasta $250 \mu \mathrm{g}$ de glucosa $\mathrm{mL}^{-1}$.

Durante el periodo de fructificación se cosecharon todos los frutos de las 10 repeticiones de cada tratamiento cuyas drupeolas estuvieran bien desarrolladas, la cosecha se realizó cuando se alcanzó el 100 \% del color negro brillante y se evaluaron las variables siguientes:

Peso fresco de fruto. Se registró el peso en g de la totalidad de frutos por repetición, con una balanza digital Ohaus ${ }^{\circledR}$ modelo Scout Pro (Parsippany-Troy Hills, New Jersey,
EUA).

Índice de redondez. En los mismos frutos que se registró el peso, se midió la longitud y diámetro ecuatorial con un vernier digital General ${ }^{\circledR}$ No. 143 (Suiza); con estos datos se calculó la relación longitud/diámetro, con lo que se obtuvo el índice de redondez, donde los valores menores a 1.0 fueron considerados como ovalados, mayores a $1.0 \mathrm{como}$ alargados y valores de 1.0 como redondos (MartínezBolaños et al., 2008).

Firmeza del fruto. Se evaluó en los mismos frutos utilizados para el índice de redondez, con un penetrómetro Qa Supplies ${ }^{\circledR}$ modelo FT O2 (Norfolk, Virginia, EUA) con punta de $2.3 \mathrm{~mm}$ de grosor a la altura del diámetro ecuatorial; los resultados se expresaron en Newtons $(\mathrm{N})$.

Sólidos solubles totales (SST, \%). Se registraron con un refractómetro digital Atago ${ }^{\circledR}$ modelo PAL-1 (Saitama, Japón) en la totalidad de los frutos obtenidos de las 10 repeticiones por tratamiento.

Acidez titulable (AT). Se utilizó la técnica descrita por la AOAC (1980); el porcentaje de acidez se calculó con base en el ácido cítrico; se seleccionaron tres frutos maduros, color negro brillante, por repetición y se determinó en cinco repeticiones por tratamiento.

Azúcares solubles totales (AST) en fruto. Se determinó con el método de antrona descrito por Witham et al. (1971), como se describió anteriormente para hojas y raíz; para ello, se seleccionaron cinco frutos maduros por tratamiento, esto se hizo en cinco repeticiones por tratamiento. Los resultados se expresaron en $\mathrm{g} 100 \mathrm{~g}^{-1}$ p.f.

\section{Determinación de compuestos antioxidantes en frutos}

Preparación del extracto metanólico. Se usaron $5 \mathrm{~g}$ de pulpa obtenida de la mezcla de 10 frutos por planta (cinco repeticiones por tratamiento) con $50 \mathrm{~mL}$ de metanol acuoso 80 \% (v/v); la mezcla se homogeneizó con agitación en un 
vórtex; posteriormente, la mezcla se colocó en sonicación por 15 min a temperatura ambiente, se dejó reposar por 24 h y se centrifugó por 10 min a 1400 × g (Chang et al., 2002).

Fenoles totales. Se usaron $250 \mu \mathrm{L}$ del sobrenadante del extracto metanólico arriba descrito y se aplicó el método de Waterman y Mole (1994). Los resultados se expresaron en $\mathrm{mg}$ equivalentes de ácido gálico por $100 \mathrm{~g}$ de peso fresco (mg EAG $100 \mathrm{~g}^{-1}$ p.f.).

Flavonoides. Con $500 \mu \mathrm{L}$ del extracto metanólico se siguió la metodología de Chang et al. (2002). Los resultados se expresaron en mg equivalentes de quercetina por $100 \mathrm{~g}$ de peso fresco (mg EQ $100 \mathrm{~g}^{-1}$ p.f.).

Antocianinas. Se aplicó la metodología de Giusti y Wrolstad (2001). La concentración se expresó en mg de antocianinas por $100 \mathrm{~g}$ de peso fresco de fruto.

Contenido de vitamina C. Se realizó con el método 967.21 de la AOAC (1995), para lo cual se pesaron $0.5 \mathrm{~g}$ de muestra fresca de fruto y se realizó en cinco repeticiones por tratamiento. Los resultados se expresaron en mg 100 $g^{-1}$ p.f.

Actividad antioxidante del fruto. Se realizó por el método ABTS, descrito por Re et al. (1999), a partir del extracto metanólico descrito previamente. Los resultados se expresaron en $\mu \mathrm{mol}$ equivalentes de trólox por $\mathrm{g}$ de peso fresco.

\section{Análisis estadístico}

Con los datos obtenidos se realizaron análisis de varianza y pruebas de medias de Tukey $(P \leq 0.05)$ mediante el programa SAS versión 9 (SAS Institute, 2002).

\section{RESULTADOS Y DISCUSIÓN}

\section{Respuesta de la planta y rendimiento}

En comparación con el testigo (2.0 dS $\left.\mathrm{m}^{-1}\right)$, el peso seco de la parte aérea (PSPA) disminuyó 27 \% con la CE de 2.6 dS $\mathrm{m}^{-1}$ y $29 \%$ con la de $3.0 \mathrm{dS} \mathrm{m}^{-1}$; el peso seco de la raíz (PSR) se redujo hasta en $36 \%$ con la CE de 2.8; el peso seco total (PST) tuvo una disminución de $30 \%$ con las conductividades de 2.4 a $3.0 \mathrm{dS} \mathrm{m}^{-1}$ (Cuadro 2). La inhibición del crecimiento $y$, en consecuencia, la disminución del peso se debe al estrés hídrico-osmótico generado por los tratamientos salinos (Munns, 2005; Munns y Tester, 2008); las modificaciones en la transpiración causadas por el exceso de sales disueltas en la solución nutritiva o suelo puede desencadenar reducción en el crecimiento por falta de absorción de agua y nutrimentos por las raíces (Parihar et al., 2015).
El rendimiento disminuyó significativamente en las plantas con conductividades eléctricas de 2.4 a 3.0 dS $\mathrm{m}^{-1}$, con una reducción de $60 \%$ con la conductividad más alta. El número de frutos por planta, en relación con el testigo, presentó una disminución del 34 al $50 \%$ con las conductividades de 2.2 a $3.0 \mathrm{dS}$, pero sin diferencias estadísticas entre ellas (Cuadro 3 ). La reducción del rendimiento y número de frutos se debe a la salinidad en la rizósfera, lo que afecta la producción de los cultivos debido al menor crecimiento de la planta, con follaje reducido $y$, por lo tanto, fisiológicamente menos activo (Parihar et al., 2015) y, en consecuencia, una reducción en la formación de los fotosintatos que la planta requiere para la producción de frutos.

La concentración de prolina en la raíz se incrementó considerablemente $(P \leq 0.01)$ con las conductividades de 2.6 a 3.0, con esta última el aumento fue de $122 \%$ en comparación con el testigo (Cuadro 3). Resultados similares se encontraron en fresa cv. Camarosa y en los genotipos chilenos Bau y Cucao (Garriga et al., 2015) y en los cultivares Camarosa y Chandler (Turhan y Eris, 2009) en respuesta a la salinidad. La acumulación de prolina en las hojas y la raíz en situaciones de estrés salino contribuye al ajuste osmótico del citoplasma celular, estabiliza y protege las estructuras subcelulares como membranas y proteínas y permite que se mantenga estable el potencial rédox celular (Parihar et al., 2015).

La longitud de la caña principal no se vio afectada por los tratamientos, pero el diámetro disminuyó en 29 y 23 $\%$ con las conductividades de 2.6, 2.8 y 3.0, sin diferencias estadísticas entre ellas (Cuadro 3). En las plantas sometidas a salinidad por $\mathrm{NaCl}$, el $\mathrm{Na}^{+}$es retenido en las vacuolas de las bases de los tallos y raíces, o a través de las células de todo el tallo, sobre todo en aquellas plantas consideradas de tallo largo, donde las altas concentraciones del ion en el xilema pueden afectar el crecimiento del mismo (Tester y Davenport, 2003).

La concentración de AST en la raíz presentó diferencias significativas $(P \leq 0.01)$ entre tratamientos, con disminución de hasta $70 \%$ en las plantas que se desarrollaron en el medio con $3.0 \mathrm{dS} \mathrm{m}^{-1}$; sin embargo, la concentración en las hojas no se afectó significativamente por los tratamientos (Cuadro 4), lo que puede deberse al consumo de los azúcares por la actividad radical para contrarrestar el estrés osmótico provocado por las conductividades eléctricas de los tratamientos, como lo es la síntesis de prolina, como se observa en el Cuadro 3. En raíces de un genotipo de arroz (Oryza sativa) sensible a salinidad (IR29) la concentración de AST disminuyó cuando el estrés salino fue prolongado (Nemati et al., 2011). 
Cuadro 2. Efecto de la CE de la solución nutritiva en el peso seco de la raíz, parte aérea y total de plantas de zarzamora cv. Tupy bajo condiciones de invernadero.

\begin{tabular}{lccc}
\hline CE $\left(\right.$ ds $\left.~^{-1}\right)$ & PSPA $(\mathrm{g})$ & PSR $(\mathrm{g})$ & PST $(\mathrm{g})$ \\
\hline 2.0 (Testigo) & $89.96 \pm 6.67 \mathrm{a}^{+}$ & $106.18 \pm 5.21 \mathrm{a}$ & $196.14 \pm 10.17 \mathrm{a}$ \\
2.2 & $81.13 \pm 4.96 \mathrm{ab}$ & $106.08 \pm 10.44 \mathrm{a}$ & $187.21 \pm 14.54 \mathrm{a}$ \\
2.4 & $66.55 \pm 3.92 \mathrm{ab}$ & $73.02 \pm 7.62 \mathrm{~b}$ & $139.57 \pm 10.33 \mathrm{~b}$ \\
2.6 & $65.53 \pm 6.06 \mathrm{~b}$ & $87.33 \pm 8.19 \mathrm{ab}$ & $152.86 \pm 14.03 \mathrm{ab}$ \\
2.8 & $68.18 \pm 5.26 \mathrm{ab}$ & $68.04 \pm 5.56 \mathrm{~b}$ & $136.22 \pm 8.9 \mathrm{~b}$ \\
3.0 & $63.56 \pm 5.19 \mathrm{~b}$ & $72.98 \pm 4.00 \mathrm{~b}$ & $136.54 \pm 8.23 \mathrm{~b}$ \\
DSH $(0.05)$ & 22.64 & 29.97 & 47.43 \\
\hline
\end{tabular}

${ }^{\top}$ Medias \pm error estándar. Medias con letras iguales en las columnas no son diferentes estadísticamente (Tukey, $\left.\mathrm{P} \leq 0.05\right)$. DSH: diferencia significativa honesta. PSPA: peso seco parte aérea; PSR: peso seco de raíz; PST: peso seco total.

Cuadro 3. Rendimiento, número de frutos, concentración de prolina y crecimiento de zarzamora cv. Tupy bajo diferentes niveles de CE en la solución nutritiva en condiciones de invernadero.

\begin{tabular}{lccccc}
\hline CE $\left(\mathrm{dS} \mathrm{m}^{-1}\right)$ & $\begin{array}{c}\text { Rendimiento } \\
\text { (g/planta) }\end{array}$ & $\begin{array}{c}\text { Núm. de frutos } \\
\text { por planta }\end{array}$ & $\begin{array}{c}\text { Prolina en raíz } \\
\left(\mathrm{mg} \mathrm{mL}^{-1}\right)\end{array}$ & $\begin{array}{c}\text { Longitud de } \\
\text { caña }(\mathrm{cm})\end{array}$ & $\begin{array}{c}\text { Diámetro de } \\
\text { caña }(\mathrm{mm})\end{array}$ \\
\hline 2.0 & $84.80 \pm 10.45 \mathrm{a}^{+}$ & $22.2 \pm 1.89 \mathrm{a}$ & $92.89 \pm 4.61 \mathrm{c}$ & $129.8 \pm 2.07 \mathrm{a}$ & $10.46 \pm 0.34 \mathrm{a}$ \\
2.2 & $61.76 \pm 6.63 \mathrm{ab}$ & $14.6 \pm 0.99 \mathrm{~b}$ & $100.50 \pm 3.37 \mathrm{c}$ & $131.4 \pm 4.67 \mathrm{a}$ & $10.34 \pm 0.42 \mathrm{a}$ \\
2.4 & $48.19 \pm 7.10 \mathrm{bc}$ & $10.9 \pm 1.08 \mathrm{~b}$ & $102.89 \pm 3.81 \mathrm{c}$ & $137.8 \pm 6.54 \mathrm{a}$ & $9.12 \pm 0.71 \mathrm{ab}$ \\
2.6 & $46.15 \pm 4.66 \mathrm{bc}$ & $12.9 \pm 0.88 \mathrm{~b}$ & $165.22 \pm 6.14 \mathrm{ab}$ & $133.8 \pm 6.44 \mathrm{a}$ & $7.44 \pm 0.34 \mathrm{~b}$ \\
2.8 & $34.72 \pm 4.00 \mathrm{c}$ & $11.1 \pm 0.86 \mathrm{~b}$ & $145.22 \pm 8.63 \mathrm{~b}$ & $125.6 \pm 4.09 \mathrm{a}$ & $8.10 \pm 0.31 \mathrm{~b}$ \\
3.0 & $33.77 \pm 2.11 \mathrm{c}$ & $10.2 \pm 0.67 \mathrm{~b}$ & $206.45 \pm 21.17 \mathrm{a}$ & $117.8 \pm 4.79 \mathrm{a}$ & $7.98 \pm 0.49 \mathrm{~b}$ \\
DSH (0.05) & 49.13 & 6.03 & 41.71 & 21.94 & 2.01 \\
\hline
\end{tabular}

${ }^{\top}$ Medias \pm error estándar. Medias con letras iguales en las columnas no son diferentes estadísticamente (Tukey, P $\left.\leq 0.05\right)$. DSH: diferencia significativa honesta.

La concentración de los pigmentos fotosintéticos se vio afectada por los tratamientos $(P \leq 0.01)$, los carotenoides disminuyeron a partir de la CE de $2.6 \mathrm{dS} \mathrm{m}^{-1}$, pero con valores similares estadísticamente con los obtenidos con 2.8 y $3.0 \mathrm{dS} \mathrm{m}^{-1}$, con una reducción de hasta $46 \%$ con la más alta CE. Las concentraciones de clorofila A (CA), B (CB) y total (CT) disminuyeron con el aumento de la salinidad, con 3.0 dS m-1 se observó la mayor reducción ( $54 \%$ de CA, $41 \%$ de CB y $50 \%$ de CT) (Cuadro 4). Resultados similares se encontraron en frambueso sometido a tratamientos con diferentes niveles de salinidad (10,20, 30 y $60 \mathrm{mM}$ de $\mathrm{NaCl}$ ) (Neocleous y Vasilakakis, 2007); lo mismo se observó en arroz (Amirjani, 2011) con estrés por $\mathrm{NaCl}$. Bajo estrés salino prolongado se acelera la senescencia de las hojas, por lo que disminuyen las concentraciones de pigmentos fotosintéticos y con ello la tasa fotosintética (Munns y Tester 2008; Parida et al., 2002). Otra explicación proporcionada por Hanafy et al. (2008) es que la biosíntesis de clorofilas podría ser inhibida por el efecto depresivo que genera la salinidad en la absorción de iones participantes en la formación del cloroplasto como el Mg y Fe.

\section{Calidad del fruto}

El peso fresco, longitud y diámetro del fruto disminuyeron $(P \leq 0.01)$ a partir de la CE de $2.6 \mathrm{dS} \mathrm{m}^{-1}$, sin diferencia estadística con las conductividades de 2.8 y $3.0 \mathrm{dS} \mathrm{m^{-1 }}$ (Cuadro 5). El índice de redondez disminuyó con las conductividades de 2.8 y $3.0 \mathrm{dS} \mathrm{m}^{-1}$, con valores registrados de 0.9 , por lo que los frutos fueron más ovalados que los obtenidos con las conductividades menores y el testigo (Cuadro 5). La firmeza se incrementó en 11 \% con la CE de $2.8 \mathrm{dS} \mathrm{m}^{-1}$; la obtenida con los demás tratamientos y el testigo fue similar estadísticamente (Cuadro 5). La disminución en el peso del fruto y firmeza por efecto de salinidad con $\mathrm{NaCl}$ también se observó en fresa Korona y Elsanta (Keutgen y Pawelzik, 2008). En el presente estudio, para alcanzar la CE deseada se incrementó paulatinamente la concentración de $\mathrm{Ca}^{2+}$, entre otros iones, conforme la CE se incrementaba (Cuadro 1); una de las funciones del $\mathrm{Ca}^{2+}$ es como elemento estructural en la pared celular, con lo que influye positivamente en la firmeza al fruto. 
Cuadro 4. Concentración de azúcares solubles totales y de pigmentos fotosintéticos en plantas de zarzamora cv. Tupy cultivada bajo diferentes niveles de CE en condiciones de invernadero.

\begin{tabular}{lcccccc}
\hline CE $\left(\mathrm{dS} \mathrm{m} \mathrm{m}^{-1}\right)$ & $\begin{array}{c}\text { Concentración } \\
\text { de AST raíz (\%) }\end{array}$ & $\begin{array}{c}\text { Concentración } \\
\text { de AST hoja }(\%)\end{array}$ & $\begin{array}{c}\text { CAR } \\
\left(\mathrm{mg} \mathrm{g}^{-1} \text { p.f. }\right)\end{array}$ & $\begin{array}{c}\text { CA } \\
\left(\mathrm{mg} \mathrm{g}^{-1} \text { p.f. }\right)\end{array}$ & $\begin{array}{c}\text { CB } \\
\left(\mathrm{mg} \mathrm{g}^{-1} \text { p.f. }\right)\end{array}$ & $\begin{array}{c}\text { CT } \\
\left(\mathrm{mg} \mathrm{g}^{-1} \text { p.f. }\right)\end{array}$ \\
\hline 2.0 & $0.37 \pm 0.01 \mathrm{a}^{+}$ & $2.02 \pm 0.08 \mathrm{a}$ & $3.92 \pm 0.09 \mathrm{a}$ & $0.63 \pm 0.02 \mathrm{a}$ & $0.29 \pm 0.02 \mathrm{a}$ & $0.92 \pm 0.03 \mathrm{a}$ \\
2.2 & $0.29 \pm 0.01 \mathrm{cb}$ & $1.96 \pm 0.06 \mathrm{a}$ & $3.60 \pm 0.10 \mathrm{a}$ & $0.54 \pm 0.01 \mathrm{~b}$ & $0.24 \pm 0.01 \mathrm{ab}$ & $0.79 \pm 0.02 \mathrm{~b}$ \\
2.4 & $0.31 \pm 0.02 \mathrm{~b}$ & $2.04 \pm 0.15 \mathrm{a}$ & $3.00 \pm 0.12 \mathrm{~b}$ & $0.42 \pm 0.02 \mathrm{c}$ & $0.24 \pm 0.01 \mathrm{bc}$ & $0.64 \pm 0.03 \mathrm{c}$ \\
2.6 & $0.23 \pm 0.01 \mathrm{~cd}$ & $2.15 \pm 0.06 \mathrm{a}$ & $2.39 \pm 0.08 \mathrm{c}$ & $0.37 \pm 0.01 \mathrm{~cd}$ & $0.21 \pm 0.01 \mathrm{ab}$ & $0.62 \pm 0.02 \mathrm{c}$ \\
2.8 & $0.22 \pm 0.02 \mathrm{~d}$ & $1.78 \pm 0.07 \mathrm{a}$ & $2.37 \pm 0.12 \mathrm{c}$ & $0.33 \pm 0.01 \mathrm{~d}$ & $0.21 \pm 0.01 \mathrm{bc}$ & $0.55 \pm 0.03 \mathrm{~cd}$ \\
3.0 & $0.11 \pm 0.00 \mathrm{e}$ & $1.95 \pm 0.08 \mathrm{a}$ & $2.12 \pm 0.04 \mathrm{c}$ & $0.29 \pm 0.01 \mathrm{~d}$ & $0.17 \pm 0.00 \mathrm{c}$ & $0.46 \pm 0.01 \mathrm{~d}$ \\
DSH (0.05) & 0.05 & 0.37 & 0.41 & 0.07 & 0.05 & 0.12 \\
\hline
\end{tabular}

${ }^{\dagger}$ Medias \pm error estándar. Medias con letras iguales en las columnas no son diferentes estadísticamente (Tukey, P $\left.\leq 0.05\right)$. AST: azúcares solubles totales, CAR: carotenoides, CA: clorofila A, CB: clorofila B, DSH: diferencia significativa honesta.

Cuadro 5. Efecto de la CE de la solución nutritiva en las características físicas de frutos de zarzamora cv. Tupy cultivada bajo condiciones de invernadero.

\begin{tabular}{lccccc}
\hline CE $\left(\mathrm{dS} \mathrm{m} \mathrm{m}^{-1}\right)$ & Peso $(\mathrm{g})$ & Longitud $(\mathrm{mm})$ & Diámetro $(\mathrm{mm})$ & Índice de redondez & Firmeza $(\mathrm{N})$ \\
\hline 2.0 & $4.00 \pm 0.10 \mathrm{a}^{+}$ & $18.32 \pm 0.26 \mathrm{a}$ & $18.58 \pm 0.18 \mathrm{a}$ & $0.99 \pm 0.01 \mathrm{a}$ & $1.84 \pm 0.02 \mathrm{~b}$ \\
2.2 & $4.37 \pm 0.10 \mathrm{a}$ & $18.90 \pm 0.29 \mathrm{a}$ & $19.02 \pm 0.18 \mathrm{a}$ & $0.99 \pm 0.08 \mathrm{a}$ & $1.92 \pm 0.04 \mathrm{ab}$ \\
2.4 & $4.30 \pm 0.14 \mathrm{a}$ & $18.61 \pm 0.37 \mathrm{a}$ & $18.64 \pm 0.23 \mathrm{a}$ & $0.99 \pm 0.01 \mathrm{a}$ & $1.98 \pm 0.04 \mathrm{ab}$ \\
2.6 & $3.57 \pm 0.12 \mathrm{~b}$ & $16.86 \pm 0.30 \mathrm{~b}$ & $17.32 \pm 0.22 \mathrm{~b}$ & $0.96 \pm 0.01 \mathrm{ab}$ & $1.94 \pm 0.03 \mathrm{ab}$ \\
2.8 & $3.22 \pm 0.10 \mathrm{~b}$ & $15.88 \pm 0.37 \mathrm{~b}$ & $17.35 \pm 0.16 \mathrm{~b}$ & $0.90 \pm 0.01 \mathrm{c}$ & $2.04 \pm 0.05 \mathrm{a}$ \\
3.0 & $3.32 \pm 0.09 \mathrm{~b}$ & $16.13 \pm 0.29 \mathrm{~b}$ & $17.20 \pm 0.20 \mathrm{~b}$ & $0.93 \pm 0.01 \mathrm{bc}$ & $1.95 \pm 0.03 \mathrm{ab}$ \\
DSH (0.05) & 0.43 & 1.14 & 0.75 & 0.05 & 0.14 \\
\hline
\end{tabular}

${ }^{\top}{ }^{\top}$ Medias \pm error estándar. Medias con letras iguales en las columnas no son diferentes estadísticamente (Tukey, P $\left.\leq 0.05\right)$. DSH: diferencia significativa honesta.

La concentración de AST presentó una tendencia a disminuir $(P \leq 0.01)$ con el aumento de la salinidad, con

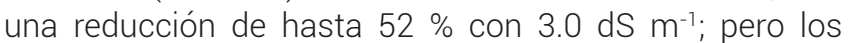
SST y la AT se incrementaron $(P \leq 0.01)$ en $8 \%$ y $22 \%$, respectivamente, con 2.8 y $3.0 \mathrm{dS} \mathrm{m}^{-1}$, sin diferencias estadísticas entre los dos tratamientos; la relación SST/ AT disminuyó $(P \leq 0.01)$ a partir de $2.6 \mathrm{dS} \mathrm{m}^{-1}$ de $C E$, el valor más bajo (11.4) se registró con $3.0 \mathrm{dS} \mathrm{m}^{-1}$ (Cuadro 6). La relación SST/AT disminuyó debido a que la AT se incrementó en mayor proporción que los SST; sin embargo, el valor de esta relación para zarzamora Tupy fue mayor en todos los tratamientos con salinidad que lo reportado para zarzamora (6.7) por De Souza et al. (2014), quienes no indican el cultivar evaluado. La disminución de AST se debe a la disminución en la actividad fotosintética de las hojas, ya que los pigmentos fotosintéticos disminuyeron con la salinidad (Cuadro 4), por lo que hubo menos fotoasimilados movilizados a los frutos. El incremento en IOS SST y AT con las altas conductividades puede estar asociado con la reducción en el contenido de agua en el fruto, ya que esos frutos también registraron menor peso (Cuadro 5), ésto posiblemente debido al aumento de $\mathrm{Na}^{+}$,
$\mathrm{Cl}^{-} \mathrm{o} \mathrm{K}$, e incluso de otros iones con los que se aumentó la salinidad (Del Amor et al., 2001).

La concentración de fenoles (Cuadro 6) y flavonoides (Cuadro 7) disminuyó $(P \leq 0.01)$ con el aumento de la CE, la mayor reducción fue de $25 \%$ en fenoles y de $29 \%$ en flavonoides con la CE de $3.0 \mathrm{dS} \mathrm{m}^{-1}$; en contraste, las antocianinas, con excepción del tratamiento con 3.0 dS $\mathrm{m}^{-1}$, cuyo valor fue similar al del testigo, se incrementaron en $95 \%$ con las conductividades de 2.2 a $2.8 \mathrm{dS} \mathrm{m}^{-1}$, sin diferencias estadísticas entre ambas (Cuadro 7); la concentración de vitamina $\mathrm{C}$, capacidad antioxidante y porcentaje de inhibición aumentaron $(P \leq 0.01)$ en 10, 21 y $18 \%$, respectivamente, con la CE de $3.0 \mathrm{dS} \mathrm{m}^{-1}$ (Cuadro 7). Estos resultados coinciden con los reportados en otros cultivos, como frambueso sometido a salinidad por $\mathrm{NaCl}$ $(10,20,30$ y $60 \mathrm{mM})$, donde la capacidad antioxidante, fenoles y vitamina $\mathrm{C}$ tuvieron incrementos con el aumento de salinidad, mientras que las antocianinas no se afectaron por los tratamientos (Neocleous y Vasilakakis, 2008). En fresa cv. Elsanta (sensible) y Korona (menos sensible) tratadas con 40 y $80 \mathrm{mmol} \mathrm{L}^{-1}$ de $\mathrm{NaCl}$ se observó 
Cuadro 6. Efecto de la CE de la solución nutritiva en las características químicas de los frutos de zarzamora cv. Tupy cultivada bajo condiciones de invernadero.

\begin{tabular}{lccccc}
\hline CE $\left(\mathrm{dS} \mathrm{m}^{-1}\right)$ & AST (g $100 \mathrm{~g}^{-1}$ p.f.) & SST (\%) & AT (\%) & Relación SST/AT & $\begin{array}{c}\text { Fenoles (mg EAG } \\
\left.100 \mathrm{~g}^{-1} \mathrm{p.f} .\right)\end{array}$ \\
\hline 2.0 & $3.81 \pm 0.27 \mathrm{a}^{+}$ & $11.26 \pm 0.15 \mathrm{~b}$ & $0.89 \pm 0.02 \mathrm{~b}$ & $12.81 \pm 0.24 \mathrm{a}$ & $644.30 \pm 5.10 \mathrm{a}$ \\
2.2 & $3.76 \pm 0.22 \mathrm{ab}$ & $11.30 \pm 0.16 \mathrm{~b}$ & $0.86 \pm 0.03 \mathrm{~b}$ & $13.38 \pm 0.20 \mathrm{a}$ & $617.76 \pm 7.19 \mathrm{ab}$ \\
2.4 & $2.87 \pm 0.13 \mathrm{bc}$ & $11.18 \pm 0.20 \mathrm{~b}$ & $0.87 \pm 0.03 \mathrm{~b}$ & $13.07 \pm 0.28 \mathrm{a}$ & $594.36 \pm 12.00 \mathrm{bc}$ \\
2.6 & $3.48 \pm 0.32 \mathrm{abc}$ & $11.88 \pm 0.19 \mathrm{ab}$ & $0.97 \pm 0.03 \mathrm{ab}$ & $12.44 \pm 0.31 \mathrm{~b}$ & $565.39 \pm 6.48 \mathrm{~cd}$ \\
2.8 & $2.78 \pm 0.15 \mathrm{c}$ & $12.51 \pm 0.25 \mathrm{a}$ & $1.04 \pm 0.05 \mathrm{a}$ & $12.14 \pm 0.26 \mathrm{~b}$ & $541.82 \pm 5.82 \mathrm{~d}$ \\
3.0 & $1.81 \pm 0.12 \mathrm{~d}$ & $12.19 \pm 0.32 \mathrm{a}$ & $1.09 \pm 0.03 \mathrm{a}$ & $11.38 \pm 0.19 \mathrm{c}$ & $484.78 \pm 7.51 \mathrm{e}$ \\
DSH (0.05) & 0.90 & 0.77 & 0.14 & 1.05 & 31.69 \\
\hline
\end{tabular}

${ }^{\dagger}$ Medias \pm error estándar. Letras iguales en las columnas no son diferentes estadísticamente (Tukey, P $\left.\leq 0.05\right)$. AST: azúcares solubles totales, SST: sólidos solubles totales, AT: acidez titulable, DSH: diferencia significativa honesta.

Cuadro 7. Efecto de la CE de la solución nutritiva en el contenido de compuestos antioxidantes y actividad antioxidante (ABTS) en frutos de zarzamora cv. Tupy cultivada bajo condiciones de invernadero.

\begin{tabular}{|c|c|c|c|c|c|}
\hline C.E. $\left(\mathrm{dS} \mathrm{m}^{-1}\right)$ & $\begin{array}{l}\text { Flavonoides } \\
\text { (mg EQ } 100 \mathrm{~g}^{-1} \text { p.f.) }\end{array}$ & $\begin{array}{l}\text { Antocianinas } \\
\text { (mg } 100 \mathrm{~g}^{-1} \text { p.f.) }\end{array}$ & $\begin{array}{c}\text { Vitamina C } \\
\text { (mg } 100 \mathrm{~g}^{-1} \text { p.f.) }\end{array}$ & $\begin{array}{l}\text { Actividad antioxidante } \\
\text { ( } \mu \text { mol E trólox g }{ }^{-1} \text { p.f.) }\end{array}$ & $\begin{array}{l}\text { Porcentaje de } \\
\text { Inhibición }\end{array}$ \\
\hline 2.0 & $37.14 \pm 2.97 \mathrm{a}^{\dagger}$ & $48.63 \pm 4.62 b$ & $19.03 \pm 0.59 b$ & $10.72 \pm 0.17 b c$ & $70.72 \pm 1.01 \mathrm{bc}$ \\
\hline 2.2 & $29.76 \pm 1.49 b$ & $95.23 \pm 4.93 \mathrm{a}$ & $20.02 \pm 0.59 a b$ & $10.35 \pm 0.15 c$ & $68.54 \pm 0.88 c$ \\
\hline 2.4 & $27.72 \pm 0.89 b$ & $96.24 \pm 3.69 \mathrm{a}$ & $20.04 \pm 0.24 a b$ & $10.15 \pm 0.26 c$ & $67.34 \pm 1.53 c$ \\
\hline 2.6 & $27.17 \pm 0.45 b$ & $93.94 \pm 5.44 \mathrm{a}$ & $19.04 \pm 0.28 b$ & $10.36 \pm 0.13 c$ & $68.60 \pm 0.75 c$ \\
\hline 2.8 & $27.16 \pm 0.73 b$ & $96.94 \pm 4.00 \mathrm{a}$ & $20.04 \pm 0.32 a b$ & $11.38 \pm 0.11 b$ & $74.58 \pm 0.63 b$ \\
\hline 3.0 & $26.17 \pm 0.89 b$ & $65.96 \pm 2.58 b$ & $20.99 \pm 0.08 a$ & $12.95 \pm 0.14 a$ & $83.84 \pm 0.84 a$ \\
\hline DSH (0.05) & 5.85 & 17.81 & 1.65 & 0.69 & 4.08 \\
\hline
\end{tabular}

${ }^{\dagger}$ Medias \pm error estándar. Letras iguales en las columnas no son diferentes estadísticamente (Tukey, P $\left.\leq 0.05\right)$. DSH: diferencia significativa honesta.

incremento en la capacidad antioxidante, contenido de fenoles y antocianinas (Keutgen y Pawelzik, 2007). Ante el estrés abiótico que implica la salinidad, las plantas expresan distintos mecanismos de detoxificación de las especies reactivas de oxígeno (ROS) en la célula, uno de ellos es la síntesis de compuestos antioxidantes como fenoles (que incluyen flavonoides y antocianinas) y ciertos ácidos orgánicos como el ácido ascórbico (Jaleel et al., 2009) que protegen a las células del estrés oxidativo que se presenta bajo condiciones de salinidad (Munns y Tester, 2008).

\section{CONCLUSIONES}

Los resultados indican que la zarzamora cv. Tupy disminuye su rendimiento y crecimiento con conductividades eléctricas en la solución nutritiva por arriba de $2.4 \mathrm{dS} \mathrm{m}^{-1}$, lo que indica que no es apta para cultivarse en ambientes salinos. Las altas conductividades eléctricas $\left(2.6,2.8\right.$ y 3.0 dS $\mathrm{m}^{-1}$ ) promovieron la acumulación de prolina en la raíz hasta en $122 \%$; así mismo, estas conductividades afectaron la calidad del fruto, en detrimento del peso, tamaño, índice de redondez y relación SST/AT, en tanto que la firmeza no se afectó con los tratamientos; sin embargo, los compuestos antioxidantes como antocianinas, vitamina C, capacidad antioxidante y porcentaje de inhibición aumentaron con las conductividades más altas.

\section{BIBLIOGRAFÍA}

Amirjani M. (2011) Effect of salinity stress on growth, sugar content pigments and enzyme activity of rice. International Journal of Botany 7:73-81, https://doi.org/10.3923/ijb.2011.73.81

AOAC, Association of Official Analytical Chemists (1980) Official Methods of Analysis. $12^{\text {th }}$ edition. Association of Official Analytical Chemists Washington, D. C., USA. 1018 p.

AOAC, Association of Official Analytical Chemists (1995) Official Methods of Analysis. 16th edition. Association of Official Analytical Chemists Washington, D. C. USA. 2490 p.

Bates L., R. P. Waldren and I. D. Teare (1973) Rapid determination of free proline for water-stress studies. Plant and Soil 39:205-207, https://doi.org/10.1007/BF00018060

Chang C. C., M. H. Yang, H. M. Wen and J. C. Chern (2002) Estimation of total flavonoid content in propolis by two complementary colorimetric methods. Journal of Food and Drug Analysis 
10:178-182, https://doi.org/10.38212/2224-6614.2748

Clark J. R. and C. E. Finn (2014) Blackberry cultivation in the world. Revista Brasileira de Fruticultura 36:46-57, https://doi. org/10.1590/0100-2945-445/13

De Souza V. R., P. A. P. Pereira, T. L. T. Da Silva, L. C. O. Lima, R. Pio and F. Queiroz (2014) Determination of the bioactive compounds, antioxidant activity and chemical composition of Brazilian blackberry, red raspberry, strawberry, blueberry and sweet cherry fruits. Food Chemistry 156:362-368, https://doi. org/10.1016/j.foodchem.2014.01.125

Del Amor F. M., V. Martínez and A. Cerdá (2001) Salt tolerance of tomato plants as affected by stage of plant development. HortScience 36:1260-1263, https://doi.org/10.21273/HORTSCI.36.7.1260

FIRA, Fideicomisos Instituidos en Relación a la Agricultura (2016) Panorama Agroalimentario. Berries 2016. Dirección de Investigación y Evaluación Económica y Sectorial, FIRA. México, D. F. 41 p.

Garriga M., C. A. Muñoz, P. D. S. Caligari and J. B. Retamales (2015) Effect of salt stress on genotypes of commercial (Fragaria $\times$ ananassa) and Chilean strawberry (F. chiloensis). Scientia Horticulturae 195:37-47, https://doi.org/10.1016/j.scienta.2015.08.036

Giusti M. M. and R. E. Wrolstad (2001) Characterization and measurement of anthocyanins by UV-visible spectroscopy. Current Protocols in Food Analytical Chemistry 00:F1.2.1-F1.2.13, https://doi. org/10.1002/0471142913.faf0102s00

González-Jiménez S. L., A. M. Castillo-González, M. R. García-Mateos, L. A. Valdez-Aguilar, C. Ybarra-Moncada y E. Avitia-García (2020) Respuesta de fresa cv. Festival a la salinidad. Revista Fitotecnia Mexicana 43:53-60, https://doi.org/10.35196/rfm.2020.1.53

Hanafy A. A., E. M. Harb, M. A. Higazy and S. H. Morgan (2008) Effect of silicon and boron foliar applications on wheat plants grown under saline soil conditions. International Journal of Agricultural Research 3:1-26, https://doi.org/10.3923/ijar.2008.1.26

Jaleel C. A., K. Riadh, R. Gopi, P. Manivannan, J. Inès, H. J. Al-Jaburi, ... and R. Panneerselvam (2009) Antioxidant defense responses: physiological plasticity in higher plants under abiotic constraints. Acta Physiologiae Plantarum 31:427-436, https://doi.org/10.1007/s11738-009-0275-6

Khan K., P. Agarwal, A. Shanware and V. A. Sane (2015) Heterologous expression of two jatropha aquaporins imparts drought and salt tolerance and improves seed viability in transgenic Arabidopsis thaliana. PLOSONE 10(6):e0128866, https://doi.org/10.1371/journal.pone.0128866

Keutgen A. and E. Pawelzik (2007) Modifications of taste-relevant compounds in strawberry fruit under $\mathrm{NaCl}$ salinity. Food Chemistry 105:14871494, https://doi.org/10.1016/j.foodchem.2007.05.033

Keutgen A. J. and E. Pawelzik (2008) Quality and nutritional value of strawberry fruit under long term salt stress. Food Chemistry 107:1413-1420, https://doi.org/10.1016/j.foodchem.2007.09.071

Kotuby-Amacher J., R. Koenig and B. Kitchen (2000) Salinity and plant tolerance. Paper 43. Utah State University Extension. Logan, Utah, USA. 8 p.

Martínez-Bolaños M., D. Nieto-Angel, D. Téliz-Ortiz, J. Rodríguez-Alcazar, M. T. Martínez-Damian, H. Vaquera-Huerta y 0. Carrillo-Mendoza (2008) Comparación cualitativa de fresas (Fragaria x ananassa Duch.) de cultivares mexicanos y estadounidenses. Revista Chapingo Serie Horticultura 14:113-119.

Munns R. (2005) Genes and salt tolerance: bringing them together. New Phytologist 167:645-663, https://doi.org/10.1111/j.1469$8137.2005 .01487 . x$

Munns R. and M. Tester (2008) Mechanisms of salinity tolerance. Annual Review of Plant Biology 59:651-681, https://doi.org/10.1146/ annurev.arplant.59.032607.092911

Nemati I., F. Moradi, S. Gholizadeh, M. A. Esmaeili and M. R. Bihamta (2011) The effect of salinity stress on ions and soluble sugars distribution in leaves, leaf sheaths and roots of rice (Oryza sativa L.) seedlings. Plant, Soil and Environment 57:26-33, https://doi.org/10.17221/71/2010-PSE

Neocleous D. and M. Vasilakakis (2007) Effects of $\mathrm{NaCl}$ stress on red raspberry (Rubus idaeus L. 'Autumn Bliss'). Scientia Horticulturae 112:282-289, https://doi.org/10.1016/j. scienta.2006.12.025

Neocleous D. and M. Vasilakakis (2008) Fruit quality of red raspberry as affected by salinity. European Journal of Horticultural Science 73:131-137

Parida A., A. B. Das and P. Das (2002) $\mathrm{NaCl}$ stress causes changes in photosynthetic pigments, proteins, and other metabolic components in the leaves of a true mangrove, Bruguiera parviflora, in hydroponic cultures. Journal of Plant Biology 45:28-36, https://doi.org/10.1007/BF03030429

Parihar P., S. Singh, R. Singh, V. P. Singh and S. M. Prasad (2015) Effect of salinity stress on plants and its tolerance strategies: a review. Environmental Science and Pollution Research 22:4056-4075, https://doi.org/10.1007/s11356-014-3739-1

Re R., N. Pellegrini, A. Proteggente, A. Pannala, M. Yang and C. Rice-Evans (1999) Antioxidant activity applying an improved ABTS radical cation decolorization assay. Free Radical Biology and Medicine 26:1231-1237, https://doi.org/10.1016/S08915849(98)00315-3

Ríos-Gómez R., C. E. Salas-García, A. Monroy-Ata and E. Solano (2010) Salinity effect on Prosopis laevigata seedlings. Terra Latinoamericana 28:99-107

Ruiz C. E., R. A. Aldaco N., J. A. Montemayor T., M. Fortis H., J. Olague R. y J. C. Villagómez G. (2007) Aprovechamiento y mejoramiento de un suelo salino mediante el cultivo de pastos forrajeros. Técnica Pecuaria en México 45:19-24

SAS Institute (2002) SAS/STAT. User's Guide. Release 9.0. SAS Institute Inc., Cary, North Carolina, USA. 4424 p.

SIAP, Servicio de Información Agroalimentaria y Pesquera (2020) Avance de siembras y cosechas. Resumen nacional por cultivo. Servicio de Información Agroalimentaria y Pesquera, Secretaría de Agricultura y Desarrollo Rural. Ciudad de México. http://infosiap.siap.gob.mx:8080/agricola_siap_gobmx/ AvanceNacionalSinPrograma.do (Junio 2020)

Strik B. C., J. R. Clark, C. E. Finn and M. P. Bañados (2007) Worldwide blackberry production. HortTechnology 17:205-213, https://doi org/10.21273/HORTTECH.17.2.205

Tester M. and R. Davenport (2003) $\mathrm{Na}^{+}$tolerance and $\mathrm{Na}^{+}$transport in higher plants. Annals of Botany 91:503-527, https://doi org/10.1093/aob/mcg058

Turhan E. and A. Eris (2009) Changes of growth, amino acids, and ionic composition in strawberry plants under salt stress conditions. Communications in Soil Science and Plant Analysis 40:33083322, https://doi.org/10.1080/00103620903325927

Waterman P. G. and S. Mole (1994) Analysis of Phenolic Plant Metabolites. Blackwell Scientific Publications. Oxford, U.K. 238 p.

Witham F. H., D. F. Blaydes and R. M. Devlin (1971) Experiments in Plant Physiology. Van Nostrand Reinhold Company. New York, USA. $254 \mathrm{p}$

Zhao Y. (2007) Berry Fruit. Value-Added Products for Health Promotion. CRC Press. Boca Ratón, Florida, USA. 442 p. 